Volume $10 \mid 2012$

Habiter : l'ancrage territorial comme support d'éducation à l'environnemental

\title{
Habiter par corps
}

Dominique Cottereau

\section{(2) OpenEdition}

Journals

Édition électronique

URL : http://journals.openedition.org/ere/1023

DOI : $10.4000 /$ ere. 1023

ISSN : 2561-2271

Éditeur

Centr'ERE

Référence électronique

Dominique Cottereau, « Habiter par corps », Éducation relative à l'environnement [En ligne], Volume 10 | 2012, mis en ligne le 20 décembre 2012, consulté le 21 février 2020. URL : http://

journals.openedition.org/ere/1023; DOI : 10.4000/ere.1023 


\title{
Habiter par corps
}

\author{
Dominique Cottereau
}

1 Apprendre à habiter par corps est au cœur des problématiques d'éducation à l'environnement. On peut bien sûr décliner une didactique environnementale composée de savoirs intellectuels, où les bénéficiaires seraient assis derrière un bureau, écoutant un exposé magistral sur le réchauffement climatique, faisant des recherches livresques sur les écosystèmes à préserver, regardant un film sur les filières des déchets. En revanche, on ne pourra concevoir une éducation à l'habiter sans la convocation plus active des corps.

2 Si je dis habiter un espace, c'est que je l'ai arpenté en long, en large et en travers. J'en reconnais les odeurs, les sons, les matières. J'en décrypte les signes, les codes, les manières d'y être. Je sais, sans le réfléchir, où mettre le pied, où changer de direction, quand tourner la tête. Je sais à quelle heure il s'emplit d'une foule pressée, et se désemplit laissant place aux courants d'air et aux changements de lumière. J'en partage les «arts de faire » et leurs ruses, braconnages et autres improvisations (de Certeau, 1990). Si j'affirme habiter un espace, c'est que je me le suis incorporé, sensiblement, par les sens et le mouvement. Nous reconnaissons avec Anne Sauvageot que «sentir, se mouvoir et s'émouvoir sont au cœur de l'action» $(2003$, p. 1). On pourrait y rajouter que sentir, se mouvoir et s'émouvoir sont au cœur de l'habiter.

3 C'est donc le point de vue du corps que je vais adopter et valoriser dans cet article. Trop souvent maintenu inactif, immobile, enfermé, dans une pédagogie dite « environnementale », le corps est pourtant le médiateur premier de nos rapports au monde, celui par lequel nous apprenons à nous mouvoir dans l'existence terrestre, celui avec lequel nous laissons notre empreinte dans l'espace. Tout comme nous n'apprenons pas à marcher par une leçon sur les lois de l'équilibre, nous n'apprenons pas à être au monde par un enseignement sur la géographie, l'écologie ou la sociologie.

4 J'illustrerai mon propos par des éléments émergeant d'une recherche-action que j'ai accompagnée, mandatée par le CIRPP (Centre d'Innovation et de Recherche en Pédagogie de Paris), au sein de l'école de TECOMAH à Jouy en Josas. De jeunes étudiants candidats au Brevet de technicien supérieur (BTS) ${ }^{1}$ en aménagement paysager y pratiquent le land art, avec pour objectif, notamment, d'apprendre à interpréter le 
milieu et à l'aménager dans le respect de ce qu'il évoque. C'est l'équipe enseignante de TECOMAH qui a proposé le projet de recherche au CIRPP. L'étude avait pour objectifs d'explorer ce qui se joue dans l'action, d'évaluer les effets de la démarche pédagogique, d'améliorer le processus de formalisation de cette démarche par les étudiants. Cette recherche heuristique nous a permis de découvrir combien les productions étaient dépendantes de la saisie corporelle du lieu. Je commencerai par poser la place du corps dans la constitution de l'être-au-monde d'un point de vue général. Je décrirai ensuite les « rayons de monde » sensoriels (Merleau-Ponty) révélés par la recherche-action, avant de témoigner de l'évolution des œuvres dans la rencontre interprétative de l'espace.

\section{Le corps, pivot de l'être-au-monde}

5 Nous naissons quelque part : lieu indifférencié. Pas de distance initiale entre le moi et le non moi - fusion. La peau frontière n'est pas encore une limite. Le corps, plastique, malléable, prêt à recevoir les signes du monde, va se charger de dessiner les limites, le dedans, le dehors. Il va apprendre à dissocier le doux et le dur, le chant et le cri, la caresse et le coup, le bleu et le rouge, le sucré et le salé, le supportable et l'insupportable. Les mots vont s'y adjoindre, mais plus tard. Le monde se donne d'abord sous la forme du sensible. Notre condition est corporelle. « Il n'est rien dans l'esprit qui n'ait d'abord séjourné dans les sens » (Le Breton, 2006, p. 25).

6 Le sentiment d'habiter vient avec la familiarité. «C'est l'appropriation qui décide de l'habitabilité, ou non, d'un lieu» (Paquot, 2006, p. 92), qui contribue à l'identité de l'individu et procure un sentiment de sécurité (Moser, 2009, p. 78). Les gestes répétés, les codes décryptés, les repères incorporés. Tout n'est qu'aisance et reconnaissance, attachement et enracinement. Nous déambulons dans nos lieux de vie quotidienne comme des poissons nageant dans l'eau. La grammaire n'est plus à déchiffrer, elle roule sous les doigts comme sous la langue, innerve chaque cellule de la peau et baigne l'oreille interne. On sait le lieu et on sait y être. «Nous devons retenir l'idée que les choses pèsent leur propre poids " écrit Pierre Sansot (2004, p. 29). Et tout ceci est irréfléchi car « entre la chair de l'homme et la chair du monde, nulle rupture, mais une continuité sensorielle toujours présente» (Le Breton, 2006, p. 13). Jeu infini des sens mêlés les uns aux autres, se répondant, se confortant, s'associant de façon confuse pour nous traduire l'espace et nous dicter notre façon d'y être.

7 Les sens sont nos outils à fabriquer du sens. Entre les sens et le sens, l'esprit fait un travail de configuration. Les perceptions, les gestes et les mouvements s'intériorisent dans la psyché sous forme de schèmes qui vont eux-mêmes organiser les représentations mentales, comme l'avait déjà expliqué Jean Piaget (1976). Lorsque je reconnais une partie du monde, je peux le faire car d'abord je l'ai vécu sensiblement et qu'ensuite j'ai organisé ces informations sensorielles dans le champ de mes représentations en une sphère signifiante d'images. Le jeu des interactions permanentes entre moi et le monde me permet d'élaborer, toujours plus densément mais aussi relativement, une pensée et des gestes sur le monde. Le corps, lieu d'accueil et d'échanges avec le réel, produit un monde d'images, lui-même organisateur du monde intelligible (Wunenburger, 1997).

8 Corps médiateur, interprète et messager, "le corps est mon être au monde, à la fois incorporation du monde et incarnation dans le monde»(Brohm, 2001, p. 108). Mon 
corps est le lieu qui donne signification à tous les lieux que je vais rencontrer. Même branché sur des machines, même prolongé d'outils ou de techniques, c'est toujours lui l'instance suprême grâce à laquelle je me coule dans le monde. Les médias qui cherchent à reproduire la multimodalité le savent, même si leurs techniques et leurs structures n'y donnent pas accès. La radio va tenter, par une mise en scène sonore, d'éveiller les autres sens, la télévision fait de même dans son jeu visuel et auditif (Sauvageot, 2003, p.69). Même les artistes du virtuel inventent de nouveaux sensoriums qui continuent de transformer les modalités sensorielles de nos sociétés (ibid., 3e partie). Car si le corps est le chemin de l'ouverture au monde, une des limites en est ce que la culture en fait (Le Breton, 2006, p. 26). Le bain culturel dans lequel nous sommes immergés, au travers l'éducation, le travail, les habitudes, ou les discours convenus, conforme autant nos représentations que nos rapports au monde. C'est aussi ce qu'ont découvert les enseignants de l'école de TECOMAH, chez leurs étudiants de BTS.

\section{Du land art pour se frotter au réel}

9 La Chambre de commerce et de l'industrie de Paris, engagée dans un important chantier de réflexion sur ses missions éducatives, a créé, en 2008, le CIRPP. Véritable laboratoire de recherche, ses finalités sont de «faire émerger, soutenir et valoriser des programmes d'études et de recherche en pédagogie, avec l'ambition de répondre à la question suivante : quels profils d'individus voulons-nous contribuer à former dans nos écoles, et selon la réponse, avec quelle(s) pédagogie(s)?». TECOMAH, aussi appelée École de l'environnement et du cadre de vie, est l'un des onze établissements inscrits dans cette démarche. TECOMAH a la particularité, installée dans une petite commune verdoyante des Yvelines, de proposer des formations environnementales: aménagement paysager, gestion et maîtrise de l'eau, horticulture, art floral, travaux publics, agro-ressources et agro-environnement.

10 En parallèle aux enseignements disciplinaires, les enseignants disposent d'un certain nombre d'heures pour monter des projets pédagogiques pluridisciplinaires. C'est dans ce cadre que trois d'entre eux, professeurs de biologie et d'aménagement paysager Corinne Meynial, Fabienne Kagan et Olivier Berger -, se sont regroupés pour concevoir un programme intitulé " art in situ ». Ils ont entrepris ce projet parce qu'ils déploraient un blocage de la créativité chez les étudiants en matière de conception de projets d'aménagement paysager. Alors que le métier demande non seulement des compétences techniques et biologiques, mais aussi une écoute sensible des lieux, une interprétation subjective argumentée et une imagination créatrice, les jeunes faisaient montre de propositions stéréotypées, conformistes, conditionnées par des années de soumission scolaire.

11 Le paysagiste est celui qui modèle les espaces de vie avec pour ambition de les embellir. Pourtant, les professeurs relataient « une grande difficulté pour les étudiants d'aborder les choses de façon personnelle et sensitive, autonome, sans passer par un contenu «scolaire » " :

Ceci entraîne tout naturellement une difficulté à oser exprimer leur perception et leur opinion personnelle. Ces difficultés dans l'approche directe de l'espace, dans la prise en compte de leur perception des formes plastiques, compliquent énormément l'apprentissage de la pratique de projets d'aménagements. En effet, ceux-ci requièrent une analyse du site qui exige un regard personnel, une 
argumentation qui exige l'expression de son regard et de son opinion sur le site. Il faut faire confiance à ses sens, oser exprimer sa perception et la confronter à celle des autres, baser une recherche sur ces pistes.

(1995), lorsque ces jeunes arrivent en BTS (ils ont entre 18 et 20 ans), ils ont à peine expérimenté cette relation à l'espace au sein de l'institution scolaire. Le temps de l'école est un temps intramuros. Pour causes de complications règlementaires, d'une demande de sécurité démesurée, d'une surcharge de programmes à faire absorber, les sorties hors les murs se réduisent d'année en année. Même si ces étudiants de TECOMAH viennent en partie de baccalauréats professionnels (travaux paysagers, forestiers, horticulture, agronomie, agriculture), où certains cours s'effectuent dehors, en direct avec l'espace, la pédagogie ne favorise ni l'apprentissage du sensible, ni l'expression de sa subjectivité. L'école transmet la distanciation plutôt que l'implication, l'objectivation plutôt que l'engagement. Si on rajoute à cela la période adolescente qui se coule sans détour dans la culture du virtuel, on commence l'inventaire des multiples freins qui font dire aux enseignants « nous perdons beaucoup de temps à «déconditionner» le regard des jeunes, à les remettre en lien direct et autonome avec une réalité perçue globalement ».

13 Ces constats ont alors amené les enseignants à intégrer la pratique du land art dans la formation. Reconnecter les élèves au réel, débrider leur imagination, expérimenter une forme d'intervention de plus en plus répandue dans l'aménagement paysager, constituent les trois objectifs du programme de pratique du land art. Ce dernier est étalé sur les deux années de BTS et sur soixante-dix heures de « cours » au total.

Pendant la première année, les étudiants découvrent l'art contemporain au travers la visite de sites et l'analyse d'œuvres. Ils sont invités à développer une approche personnelle vis-à-vis de cette forme d'art, à approfondir leur observation et leur curiosité, à s'ouvrir et sortir de leurs jugements, à bâtir une réflexion jusqu'à interpréter le propos de l'artiste, et enfin à exprimer une opinion argumentée. L'important est l'engagement et la personnalisation de la réflexion exclusivement appuyée sur leur propre perception, excluant au départ toute approche de compilation livresque ou scolaire. Ils ont ainsi l'occasion de s'approprier l'art contemporain à partir d'œuvres telles que le Cyclop de Jean Tinguely, l'Atelier de Brancusi, les Colonnes de Buren, de sites tels que Chamarande, la Défense, ou encore d'œuvres photographiées (repérées dans des ouvrages spécialisés). Dès le début de la seconde année, les enseignants leur proposent de créer une œuvre d'art en groupe, qui est, en fin de production, présentée à un public. La création a lieu dans le parc de Jouy, site de l'école et est soutenue par l'encadrement de deux artistes qui aident les jeunes à mûrir leur projet, Gilles Bruni et Paul Jacob. Un thème est défini tous les ans à partir duquel ils vont donner libre cours à leur imagination tout en s'appuyant sur le site, ses richesses, ses contraintes, ses évocations.

15 Le land art est défini comme étant une «tendance de l'art contemporain apparue aux États-Unis vers 1967 et caractérisée par un travail dans et sur la nature » (Larousse, 2011, p. 577). Sortir des musées et des galeries d'art était l'ambition première de ces artistes qui sont allés travailler in situ avec la matière brute de la nature, ses formes, ses textures, ses couleurs, ses matériaux, sa densité. Certains chantiers sont gigantesques, déplaçant des tonnes de roches (comme Heizer), peignant des pans de montagne entiers (comme Verame). D'autres sont plus à la mesure de l'homme (Goldsworthy).

Éducation relative à l'environnement, Volume 10 | 2012 
Certains amènent des objets extérieurs (Christo et Jeanne-Claude), d'autres se contentent des matériaux de la place (Nils Udo). Certains ne font pas grand cas de la préservation du lieu, usant de produits polluants ; d'autres font des œuvres éphémères, disparaissant aux premières fontes des glaces ou au souffle du vent, seuls en témoignent une photographie, un film, ou des croquis. Le land art c'est tantôt parler de l'homme à l'aide de l'art dans la nature, tantôt parler de la nature perçue par l'homme à l'aide de l'art, tantôt parler de l'art pensé par l'homme au moyen de la nature. Le land art n'est donc pas, en soi, environnementaliste ou écologique. On y retrouve toutes les façons d'aménager la nature à la convenance humaine, de la domination surpuissante à l'écoute sensible, de la transformation agressive au surlignage provisoire. Chaque artiste traduit ainsi sa façon de penser et d'habiter le monde. La démarche de TECOMAH n'a pas pour ambition de former des artistes en land art, mais plutôt de les aider à « ouvrir leurs pratiques sur un univers plus large » comme le dit Gilles Bruni, artiste accompagnateur. Apprendre à « penser par les sens » et trouver dans « la raison du corps » (Godon, 2008, p. 60) le sens d'un paysage et d'une intervention sur celui-ci sont les deux objectifs centraux du programme pédagogique.

Cette recherche-action s'est déroulée de septembre 2009 à octobre 2010. La posture du chercheur que j'y occupais ne fut pas celle, surplombante, de celui qui sait et montre la voie à ceux qui ne savent pas. Tout au contraire, il m'a d'abord fallu prendre connaissance du contexte. Les enseignants avaient beaucoup d'avance sur moi. Ils sont à l'origine de l'action, ils la mènent depuis de nombreuses années, et sont donc imprégnés de cette connaissance expérientielle, dont une partie seulement s'exprime par les mots. Notre méthode a d'abord été d'être sans méthode : je devais m'immerger dans le sujet pour en saisir le sens. Je nous devais d'abord d'être là (le «Dasein » de Morin et Kern, 1993), sans a priori, sans projection rapide. Être juste là pour écouter, regarder, sentir et "comprendre ", ce que je fis pendant six mois. Nos réunions de réflexion sur les différentes dimensions du projet se déroulèrent presqu'à bâtons rompus : je cherchais à tirer les fils de leurs doutes, de leurs besoins, mais aussi de leurs certitudes. Je suivis plusieurs séances pédagogiques, en profitant pour interroger les élèves et avoir leurs sentiments sur l'expérience qu'ils étaient en train de mener. En 2010, nous avons pu poser des axes de réflexion conceptuelle et monter un protocole d'enquête invitant les élèves à expliciter l'évolution de leur démarche tout au long des productions.

Une première demi-journée, au mois de mai, servait d'immersion dans le parc. Ils devaient, librement, se promener, seul ou en compagnie, pour choisir un lieu, le lieu qui les «touche ", leur " parle », le lieu qui pourrait répondre à la future consigne qui sera l'invite de la création: "échelle humaine». Ils eurent à regarder, ressentir, interpréter, choisir, puis dessiner ce lieu, le croquer et le commenter. Premier travail du paysagiste: observer, ressentir, consigner. La suite se réalisa après les congés scolaires de l'été, au mois de septembre. Par site choisi, ils se regroupèrent, définirent ensemble un projet et, pendant trois jours, par tâtonnement, triturations, tentatives, tests, ils habillèrent, soulignèrent ou modifièrent le lieu jusqu'à pouvoir le présenter lors d'une dernière après-midi devant les équipes de direction, d'enseignement ainsi que les étudiants de BTS en première année. Au final, 59 étudiants se répartirent en 15 groupes dans le parc de TECOMAH, véritable défi pour les artistes pédagogues qui coururent de groupe en groupe tous ces jours de création, devant trouver le bon dosage temporel d'attention à chacun tout en restant équitable envers tous. 


\section{Descendre au parc : itinérance corporelle} corporelle de cette première demi-journée de printemps. Il s'agit là de révéler combien «le réel, et, par-là, nous entendons tout ce que nous pouvons rencontrer selon les diverses modalités de l'expérience humaine, a quelque chose à nous apprendre" (Sansot, 2004, p. 57), et combien ce quelque chose passe par les sens. Commençons par le mouvement: pour entrer dans le parc de Jouy, nous empruntons une petite route bitumée qui s'enfonce sous les arbres, interdite aux voitures par une barrière que nous contournons. Je suis avec trois des étudiants, qui, après avoir reçu les consignes de travail, sont partis plus rapidement que les autres, comme désireux de perdre le moins de temps possible. Ils marchent à vive allure, sachant exactement où ils vont, familiers des lieux. La voie est bordée de grands arbres : chênes, érables, hêtres, platanes, pins... qui font lever la tête des trois garçons et redresser les corps. Le pied est sûr de lui, mais la main traîne, caressant feuilles et fougères sur son passage. L'impulsion nous porte. Pas besoin d'avoir les yeux fixés sur le sol pour nous assurer le pas, les capteurs de nos corps, dissimulés dans les muscles, les articulations et l'oreille interne, nous traduisent la pente, et nous renseignent sur la position que nous devons adopter pour progresser sans risque. Nous devons pour cela nous servir aussi de nos bras, nous balançant au rythme du pied devant l'autre. Bien sûr, nous ne mesurons pas cet enchaînement de mouvements. Il se fait spontanément, avec le minimum de vigilance requis. L'acquisition de la marche s'obtient très tôt dans les apprentissages de l'enfant, et, sauf accident, de façon irréversible. La station debout est le propre de l'humanité. C'est à partir de cette verticalité que les autres sens vont se déployer.

Et notamment la vision: parce que debout, la tête, verticale, peut orienter le regard comme elle le souhaite, sans tension. Nous pouvons balayer des yeux l'ensemble du paysage et enregistrer une somme phénoménale d'informations. « Le sens de la vue, le dernier qui soit apparu chez l'homme, est aussi de beaucoup le plus complexe » (Hall, 1971, p. 87). Il peut être un moyen de communication agissant jusqu'à un kilomètre et demi de distance. Ce récepteur est d'une grande efficacité d'identification. Nous reconnaissons, au passage, car nous les avons déjà rencontrés, le noisetier charnu, le lierre enroulé, la fougère couvre-sol, le lilas blanc et l'azalée rose. Nos yeux ne s'arrêtent pas sur tout ce qui s'étale devant nous. Nous sélectionnons sans nous en rendre compte.

Un principe d'économie s'impose en effet pour ne pas être submergé d'informations, noyé dans le visible. Une reconnaissance sommaire des données de

l'environnement suffit pour s'y mouvoir sans dommage. (Le Breton, 2006, p. 75)

Nous savons alors nous orienter au milieu des choses. La vue est le sens du jugement immédiat, surtout dans notre société occidentale qui en fait son médium par excellence " permettant à l'intelligence l'apprentissage de l'identification à travers les étapes cognitives de voir, de regarder et d'observer » (Sauvageot, 2003, p. 41). En occident, la vue (et la lumière: le jour étant son milieu de prédilection) est associée à la connaissance. Bon nombre de métaphores témoignent de cette association. L'œil de la connaissance nous préserve de l'ignorance, qui à l'inverse est cécité, obscurité, aveuglement, nuit, brouillard, flou. "Vivre se suffit d'ombre, lire exige la clarté " (Serres, 1985, p. 80).

Éducation relative à l'environnement, Volume 10 | 2012 
21 D'ailleurs au travers la consigne de croquer le lieu présupposé de la création future, la vue est fortement mise à contribution. Dessiner pour apprendre à observer. Cadrer une portion de paysage et y scruter chaque objet, chaque forme et ses mouvements, la lumière qu'il reçoit, le grain de sa matière. Les étudiants vont devoir tenter de lutter contre la naïveté de ce sens (Le Breton, 2006, p. 63) : emprisonnée dans les apparences, la vue croit tout connaître de ses balayages de regard. « La vue souffre du mélange et de son évidence " nous fait remarquer Michel Serres (1985, p. 80). Le croquis oblige à l'examen plus poussé de chaque élément du paysage. Il faut observer avec attention, transformer l'observation en schème mental et la reproduire sur le carnet avec le plus de fidélité possible. Opération rapide, complexe et efficace qui semble objectiver le regard, et pourtant même là, celui-ci reste une prise subjective sur le réel.

L'œil est sans innocence, il arrive devant les choses avec une histoire, une culture, un inconscient. Il est celui d'un sujet, enraciné au corps et aux autres sens, il ne reflète pas le monde, il le construit par ses représentations. (Le Breton, 2006, p. 82)

En dessinant, les jeunes s'approprient l'espace choisi, les autres sens ont le temps de tisser leur propre image avec l'image visuelle. Margaux, assise dans l'herbe mouillée, est totalement absorbée par la saisie d'un platane plus que centenaire. Son corps est penché du même côté qu'une des grosses branches qu'elle est en train de dessiner, ses cheveux longs tombant en miroir du feuillage. Malgré l'atmosphère brumeuse et fraîche de ce matin de mai, c'est comme si elle était oublieuse du froid et de l'humide. La nature enveloppante est malgré tout au printemps : les feuilles des arbres sont du vert tendre qui succède leur éclosion, les hirondelles survolent bruyamment l'étang au bord duquel elle se tient, une ribambelle de canetons nagent et s'époumonent derrière la canne col vert, du blanc, du rose, du jaune, du mauve, du orange transparaissent dans la végétation alentour, émanant d'odeurs suaves et boisées, tout cela Margaux le perçoit. «C'est parce que l'œil passe sans cesse de l'objet à ce qui l'entoure, et inversement de ce qui l'entoure à l'objet que celui-ci peut être vu» (Roustang, 2008, p. 162).

23 Même si nous faisons ici l'exercice de décomposer les sens pour mieux les comprendre dans leur tâche d'appropriation et d'interprétation du monde, ils sont en permanence interreliés. Nous regardons, entendons, sentons dans le même mouvement de captation de ce qui nous environne. Une information sensorielle peut dominer toutes les autres un bruit infernal, un spectacle inhabituel, une odeur répugnante, une température excessive -, elle attire alors davantage notre attention, mais les autres sont là pour nous donner le sentiment général du lieu.

Patrice, qui est assis, contemplatif, sur le rebord de l'étang, adossé à la digue, me dit «j'adore être là, profiter du dehors ». Lorsqu'on parle du sens du toucher, on oublie que c'est lui qui nous enveloppe en premier lieu. C'est ce sens tactile qui nous donne la température de l'air, son hygrométrie, son mouvement. Tiédeur de l'été ou morsure de l'hiver, sècheresse du vent d'est ou humidité du vent d'ouest, nous sommes en permanence dans des corps-à-corps avec le monde alentour, emmaillotés dans la densité du réel. Le sensible c'est d'abord la tactilité des choses, le contact avec les autres, les objets et l'air, l'intimité qu'on leur accorde. Le toucher est le sens de la plus grande réciprocité : " toucher c'est se toucher, disait Merleau-Ponty, les choses sont le prolongement de mon corps et mon corps est le prolongement du monde qui m'entoure » (Le Breton, 2006, p. 178). 
Les travaux de Hall (1971) sur la proxémie ont bien montré comment nous nous construisions des bulles invisibles protégeant notre moi d'une trop grande proximité avec les autres. Les quatre distances - intime, personnelle, sociale et publique, avec pour chacune un mode proche et un mode lointain, - organisent le degré de toucher que nous acceptons avec les individus qui nous entourent. Se toucher va au-delà du contact direct, la peau possède ses extensions subjectives qui font qu'on se laisse caresser par un être aimé, mais qu'à dix mètres, la présence d'un être détesté est encore trop "palpable». Les travaux de Montagu (1979), qui montrent combien les contacts premiers de la mère au bébé conditionnent la vie affective et psychique de l'enfant et plus tard de l'adulte, sont également reconnus. Et il y aurait bien d'autres auteurs à citer (dont Anzieu et Prayez).

La disparition du toucher est une privation de la jouissance du monde. Les choses nous baignent des pieds à la tête et si nous perdons ce sens nous perdons aussi le sens de notre présence, nous dissolvant dans l'espace, sans limite autour de nous, sans passage entre le moi et le non moi. « Le toucher est la souche fondatrice du rapport de l'homme au monde » (Le Breton, 2006, p. 177).

La plupart des étudiants que je croise dans le parc se contente de toucher du regard et de baigner dans le lieu. Matthieu, lui, ne peut s'en satisfaire. Il prend le temps, avant de réfléchir au projet de création, de s'arrêter près des serres, son lieu préféré. Et là le regard n'y suffit plus. Il se penche vers les boutures, touche chaque feuille, palpe chaque branchage. Il ramasse une fine brindille de rosier tombée, sans doute antérieurement cassée par un mouvement brusque, cherche un pot, de la terre, de l'eau. Il hume les plantes à fleurs, les unes après les autres. "Un principe anthropologique de l'odeur est de se donner comme révélateur d'une intériorité ", nous dit David Le Breton (2006, p. 253). Matthieu n'a de cesse de retrouver les correspondances entre les fragrances mélangées, détachées de leur source, les odeurs flottant dans l'air. À qui ce parfum citronné ? À qui cette exhalaison poivrée ? Et celleci, vanillée ? À chaque végétal son arôme, qui s'échappe, se diffuse, se répand, plus ou moins loin selon les vents, déterminant l'ambiance affective de l'espace. Les odeurs sont de puissants marqueurs d'atmosphère. Les mauvaises font fuir, les bonnes attirent. Elles impressionnent l'espace d'une morale aérienne, incitant à l'abandon ou à la méfiance, induisant inquiétude ou détente (ibid., p. 255).

Les odeurs sont pourtant difficiles à nommer, alors on use de métaphores et d'analogies, d'emprunts aux autres sens, on tourne autour, on associe, on digresse. "Les odeurs n'accèdent pas plus que les goûts au niveau symbolique » (Sauvageot, 2003, p.59). On nomme la source de l'odeur, mais pas l'odeur elle-même. Elles n'en demeurent pas moins une des composantes majeures de nos imaginaires, et particulièrement de nos mémoires. "Veilleuse dans la chambre des souvenirs ", comme le disait Gaston Bachelard (1989, p.122), l'odeur possède une forte puissance d'évocation. La mémoire olfactive est dense et de longue durée. Lorsqu'une circonstance nous replonge dans une odeur familière, mais ancienne, c'est un univers tout entier qui resurgit, une bribe de notre histoire où personnages, lieux, choses, mouvements reprennent vie. "L'odeur déplie le temps» (Le Breton, 2006, p. 267). Matthieu se fait des réserves de souvenirs. Et il n'est pas le seul. La brume qui nous enveloppe ce matin-là est un diffuseur de parfum, du parfum particulier de l'humide. Les étangs, la rosée, le brouillard composent ensemble le parfum aquatique issu de la 
végétation et de la terre. L'odeur humide donne l'âme du jour, incitant à la lenteur, au repos, au silence.

Le silence va bien à la brume. Ou bien est-ce la brume qui impose le silence? Sans doute un peu des deux. En fait, il ne s'agit pas d'un silence total. Quand on nomme le silence aujourd'hui, on ne fait que relativiser le degré sonore de nos vies quotidiennes. Il en va du silence comme du vide : il est inexistant en nos contrées terrestres. Même dans la grotte la plus profonde, et dans l'immobilité la plus totale, on entend encore les bruits de notre propre corps.

La première source du bruit gît dans l'organisme, dont l'oreille proprioceptive écoute, en vain parfois, le murmure subliminaire: des milliards de cellules s'adonnent à une action biochimique telle que nous devrions nous évanouir sous la pression de leur rumeur. (Serres, 1985, p. 134)

Les bruits du monde social sont devenus si prégnants que leur absence est connotée de silence. Et pourtant, craquement des feuilles sous le pas, sifflement des colverts, gazouillis des hirondelles, bruissement des becs d'oiseaux fouilleurs de fourrés, murmure du cours d'eau, jacassements d'une pie... univers acoustique du parc qui participe à son écologie. Le bruit est la pollution du son. Notre oreille ne peut se fermer comme les yeux, le tintamarre ne peut s'éviter comme le contact. L'ouïe n'est pas malléable, elle est obligée. On semble s'adapter, on ne fait que s'habituer, mais le corps peut en être agressé.

Plusieurs des jeunes étudiants ont choisi de s'installer auprès de la chute d'eau pour s'y enfermer. "Ce qui m'intéresse ici, me dit l'un d'eux, c'est le mouvement, l'endroit fermé qui nous permet de mieux voir le lieu, le bruit de l'eau qui empêche d'entendre les autres bruits qui ne sont pas de la nature ». Le son occupe le volume et se répand dans le global, il donne sa tonalité à l'espace et son épaisseur sensible. L'ouïe est immersion. Les rumeurs n'ont pas de lieux, à peine émises, à peine ouïes, elles ont disparu et pourtant elles semblent installées, car répétées, insaisissables et envahissantes, imprégnant l'espace. Lorsqu'elles sont nombreuses, on ne sait d'où elles viennent ni à qui elles appartiennent, mais elles pénètrent en nous sans que nous n'y puissions rien. «L'ouïe est le sens de l'intériorité, [elle] mène le monde au cœur de soi, là où la vue le jette au dehors » (Le Breton, 2006, p. 118).

Parmi toutes ces rumeurs pénétrantes, celle de la voix humaine est celle du lien social. Les jeunes sont plusieurs au pied de la chute d'eau et l'enfermement provoqué par le lieu et le son n'impose pas la suspension des paroles. Avant de les apercevoir, je les avais entendus, clameurs joyeuses cachées des yeux, portées par le vent, m'aidant à m'orienter pour les rejoindre. Ils sont trois, semblant « s'entendre » comme larrons en foire, les mots passant de l'un à l'autre, rebondissant de bouche en oreille, et d'oreille en bouche. Sur les fils de l'oralité courent la socialité, la convivialité, la transmission, l'approfondissement de la communauté d'appartenance. Sur le fil de la voix humaine galopent chaleur, amour, tendresse aussi bien que violence, haine et mépris. Parlent-ils tous les trois de la voix de l'eau ? Non. La langue glisse ailleurs, sur d'autres terrains, et jusqu'à ce qu'ils m'aient vue (car ils ne m'avaient pas entendu arriver), leurs discours faisaient obstacle à la voix du flot. " Le groupe se clôt dans un mur compact de langue. Nul ne peut faire attention à autre chose qu'à des mots ", a expérimenté Michel Serres (1985, p. 112), "rien ne rend insensible comme la parole» (ibid., p. 68), insensible à toute autre chose qu'à ce qui se dit et à celui qui parle. Les choses ne s'entendent que 
dans le silence solitaire et dans l'attente patiente. Qui veut apprendre des choses doit d'abord se taire et rendre l'oreille active.

Il nous manquera le goût pour avaler définitivement et totalement cet espace de nos sens. Il aurait fallu que l'été nous invite à nous coucher dans les prairies sèches, et l'on aurait mâchouillé quelques herbes ou graminées, suçant la sève. Bien que rarement isolées des autres modalités sensorielles - on goûte au miel du soleil, on boit la fraîcheur de la nuit, on savoure l'ombre du hêtre pleureur - il aurait fallu passer par le jardin potager, le verger ou les abords de cantine, pour éveiller nos papilles aux images gustatives qu'ils auraient taquinées. Le goût pourtant va plus loin que l'oreille, va plus profond que le nez, dans l'intériorisation du monde, dans son incorporation et son assimilation. Le monde transite en nous par ce que nous avalons de lui et nous en gardons le meilleur comme le pire, le savoureux comme l'empoisonné.

\section{Des sens qui s'éduquent : processus d'évolution observé}

On pense que le monde s'offre à nous dans toute la sincérité de son réel. Il n'en est rien. Ni innocence, ni vérité, car nous ne sommes, nous-mêmes, ni lisses, ni vides. Des années d'enseignements abstraits et intellectuels appauvrissent le dialogue entre le monde sensible et le corps. Nos sensations et nos gestes ne sont que ce que la culture en fait. Ils ne sont en aucun cas des canaux passifs par lesquels transiteraient les informations brutes du réel. Ils ne reflètent pas le monde, ils le construisent. Il n'existe pas de naturel d'un geste ou d'une sensation. Chaque société dessine sa propre organisation sensorielle (Le Breton, 2006). L'homme ne perçoit que ce qu'il a appris à percevoir, par immersion régulière dans des lieux. L'Inuit sait parler de la neige sous de multiples formes avec un nuancier de langage qui nous échappe totalement à nous, gens des plaines tempérées, qui ne savons la percevoir que froide et blanche. L'enfant qui grandit entre l'intérieur de son appartement et l'univers clos de l'école, cour carrée, asphaltée, murs bétonnés, livres et voix, cahiers et tableaux, en guise de résumé du monde apprendra un langage sensible appauvri. La chose a été remplacée par le mot de la chose, l'organique par l'image de l'organique, le territoire par la carte, le concret par son abstraction. Anesthésie corporelle pour «mieux concentrer l'esprit ", disent nos maîtres. Il ne peut même plus y avoir d'école buissonnière puisqu'il n'y a plus de buissons, désapprouve amèrement Michel Serres (1985, p. 253). Le "sensorium" (Sauvageot, 2003, p. 60) que nous proposons à nos enfants préfère le dedans au dehors, les écrans au plein vent, la lecture au goûter, l'insipide à l'épicé.

$\mathrm{Au}$ moment de la mise en production par les étudiants, le réfléchissement entre le paysage et l'interprétation ne fut pas immédiat. L'exercice de création demande plusieurs jours. C'est pourquoi même il nécessite cet accompagnement pédagogique ferme et souple à la fois. Le site fut souvent choisi en premier lieu pour ses aspects sensibles. Et pourtant, lorsqu'ils se mettent en groupe et doivent définir leur projet, la première idée qui vient à l'esprit des jeunes est une idée abstraite, plaquée sur le site sans réellement l'épouser.

" Au départ, on pensait travailler sur la déforestation. C'est un peu l'idée du moment, on parle de développement durable... » (Thomas). L'idée vient d'ailleurs, d'une image construite en amont, image souvent intellectuelle plus que corporelle. Pour la plupart des groupes, la première interprétation qui était venue à la prise de connaissance de la 
consigne " échelle humaine » avait été la pollution et la dégradation de la nature par les hommes. Une même idée quel qu'en fut le site. Se mettre d'abord en écoute lente du lieu n'est pas un acte naturel. On projette plutôt que de laisser venir. Les artistes et enseignants eurent à faire ce travail de ralentissement des idées pour que le corps prenne le temps de l'appropriation du lieu et de ses éléments.

On essaie de ne pas faire une hutte. Ce sera bien sphérique à l'intérieur, un vrai nid. Le site naturel était en creux, ça faisait penser à un abri pour animal, mais à échelle humaine. Ambiance calme, paisible, ombragée, plus la présence des deux arbres, et parallèle à la grotte. On voudrait que le visiteur puisse profiter de l'ambiance. Au départ on était dans l'excès de déchets dans la grotte, mais trop d'idées, on est donc revenu sur le site de base. On est beaucoup plus content de ce à quoi on est arrivé finalement. Fabriquer c'est plaisant. (étudiants du groupe «le nid perché de la grotte »)

Ce ralentissement est souvent mal perçu par les étudiants :

On creuse pour rien depuis une heure! Notre idée n'est pas bonne d'après l'artiste. Il nous a parlé de calque, mais ce n'est pas naturel. Pour résumer, on a plusieurs options! C'est la cinquième fois qu'on repart de zéro! Alors la première fois ça fait mal, mais là on s'y fait. Au départ on avait la butte, mais on regardait vers le sol (...). (Marielle)

Notre culture est si peu habituée à laisser ressentir avant que de dire, à expliciter avant que d'expliquer, que les étudiants ne comprennent pas, dans un premier temps, pourquoi leur idée initiale n'est pas bonne. C'est pour pallier à cette difficulté que les enseignants avaient proposé le thème d' "échelle humaine ": éviter la démesure de transformation du site, affiner la sensibilité. Lorsque le génie du lieu parle de lui-même (et le parc de TECOMAH en est riche), il n'est pas besoin d'aller très loin pour souligner un de ses aspects.

Les jeunes n'intègrent cette notion qu'après avoir retrouvé le sens corporel de leur implication. "Il m'a fallu du temps pour comprendre que c'est le site qui donne la problématique ", avoue l'un d'eux :

On a retrouvé du poil de la bête. Les deux idées qu'on avait eues au départ sont tombées à l'eau, démâtées! On a bien rebondi avec l'idée d'origamis d'animaux pour révéler le site, sans saturer le milieu. On a été attirés par la végétation, le cadre bucolique, sauvage. La première séance on a tâtonné, puis on avait trouvé une idée, mais elle s'est faite démontée par Mme Meynial. [Pendant ce premier jour de création,] en plus, on était sous la pluie, une pluie fine, pénétrante... [qui redoublait le sentiment de frustration]. À $10 \mathrm{~h}$, les artistes arrivent et pouf! Tout tombe. On a tout arrêté et on ne s'y est remis que l'après-midi (...). J'étais au bord de l'abandon. (Kim)

Un groupe s'est même saisi de cette tension dépressive pour laisser venir la nouvelle proposition: "c'est désespérant, on devrait se pendre", dit l'un d'eux lors du débriefing du second matin, leur platane d'investigation en tête. Paul, l'artiste, réagit immédiatement, "c'est une belle idée la pendaison». Ils se regardent, d'abord interloqués, puis leurs yeux s'illuminent. Ils sont, en effet, sous un arbre plus que centenaire offrant de belles branches suffisamment élevées au-dessus du sol pour jouer avec cette idée. Après un moment de discussion, l'un d'eux explique : "Le jeu du pendu : la vie est un jeu, les dés sont jetés ! (...) Ça va mieux maintenant. Au début on était bloqué. C'est la discussion qui a débloqué. On était tellement désespéré, qu'on a pris l'idée de la corde, c'était un jeu ». 
41 Les choses débloquent entre la troisième et la cinquième demi-journée. Certains déambulent dans le parc, regardent de droite et de gauche, rendent visite aux autres, discutent âprement tout en marchant. L'impulsion les porte. Leurs esprits fonctionnent au rythme de la marche attentive, opérant des allers-retours entre ce qu'ils perçoivent et leur projet. D’autres laissent décanter par une mise en veille pré-réfléchie du projet. "L'idée des grenouilles est venue en cours de math. Thomas aime faire des grenouilles de papier. Et puis le cerveau travaille toujours, même si on pense qu'il est en repos, il continue de travailler» (Marie). Marie n'est pas la seule à penser cela, Matthieu et Sophie, dans les serres, ont suivi le même procédé : "On a trouvé cette idée en se promenant, écrivant, se parlant. (...) En attendant que l'idée vienne, je lis mon journal. L'inconscient travaille sans cesse même quand on n'y pense pas. »

La phase suivante est une période joyeuse, légère en même temps que fort productive. Ils découpent, ramassent, portent, agencent, grimpent, courent, scient, peignent, balaient... artisans des arbres, des feuilles, de l'eau ; tantôt silencieux, tantôt bavards. Ils partagent les tâches et organisent le travail à la chaîne, s'entraidant mutuellement, avec une frénésie de faire beau et bien. "Ça va très très bien! On est motivé ce matin. (...) Nous, ça marche. On le trouve très esthétique. Ça va être sympa de présenter, de voir les avis des gens, s'ils comprennent, car c'est quand même le but " (groupe « latitude longitude »). Heureux d'être là où ils sont à faire ce qu'ils font, exposés sous le soleil (« dans la joie et la bonne humeur, en plus il fait beau!») où à l'abri dans des coins ombragés ( On fait du défrichage, c'est cool. Il y a une bonne ambiance, on est au frais» groupe "retour aux sources»). Les mains fonctionnent vite, car ils se sont approprié à la fois le site et l'idée, les deux allant bien ensemble. L'idée semble aller de soi, c'est une évidence qui déroule son sens et qui les envahit d'une certitude réconfortante:

On en est à aménager l'intérieur. On aime bien la structure comme cela, c'est plus rond. Et ce sera encore mieux quand on aura fermé la porte en bas. Pour nous c'est vraiment notre projet. On se l'approprie, d'ailleurs on va pique-niquer dedans ce midi. C'est ma première cabane, mais j'en ai toujours rêvée. C'est un espace confiné. (groupe « nid perché de la grotte »)

Presque tous les groupes sont capables, à ce moment-là, d'argumenter le choix de leur installation en dialogue avec le site :

Maintenant on a enfin notre idée. Ce matin, on était perdu. Là on a prévu un tableau dans les pétasites. Rappeler la nature de façon artificielle, tableau 18e siècle. Le choix du site : mystérieux, différent des autres paysages, avec ces grandes plantes. C'est sauvage, il y a le bruit de l'eau, c'est confiné, la végétation est dense. C'est intrigant. (groupe « le théâtre du douanier Rousseau »)

«Fier, totalement fier. Avec les feuilles c'est vraiment... ça habille. (... ) Avec notre œuvre on a beaucoup avancé, ça prend du sens. On ne pourrait pas faire ça ailleurs, c'est vraiment in situ », s'exclamera Cyril à la fin de l'installation. "Le fait qu'on nous demande de nous justifier ça nous fait avancer ", surenchérit Margaux. En effet, de justifier ses choix implique de décrire ce sur quoi s'enracine l'idée d'installation, description qui ne peut que passer par l'explicitation du sensible: ce que l'on voit, ce que l'on entend, ce que l'on goûte, ce que l'on hume, ce que l'on touche. La signification a émergé de ce dialogue entre le corps et l'esprit. 


\section{Conclusion}

La recherche-action n'avait pas pour ambition de révolutionner le programme « art in situ ", ni de régler des problèmes cruciaux comme peuvent en avoir besoin certains acteurs qui se lancent dans une recherche-action. Elle voulait surtout permettre à une équipe de se poser et de prendre un peu de distance sur un projet qu'elle mène depuis plus de dix ans. Le programme évolue en permanence, mais plus par adaptations successives aux contraintes grandissantes, que par orientation maîtrisée. «On avait la sensation d'être arrivé dans une impasse ! ", «ça marchait, mais c'était malgré nous ». Comme l'expriment les enseignants, la recherche-action a donc d'abord facilité la "conscientisation de ce qu'on fait» (Corinne Meynial). "Nous avons appris son importance pour les élèves » (Fabienne Kagan). Faire parler les élèves, être à leur écoute, dans cette attention bienveillante sur l'ensemble de leurs sentiments évolutifs, joue comme un révélateur puissant à la fois pour comprendre ce qui s'opère dans l'évolution des créations et de l'apprentissage, mais aussi comme source d'autoapprentissage. Avant d'avoir nommé les choses, on ne sait pas qu'on sait, il suffit d'une question (" comment ça va ce matin? ", " comment ça se passe pour vous? ", " où en êtes-vous dans le travail ?») pour que les mots répondant à la question se transforment en révélateurs, au sens photographique du terme. Gain de conscience sur soi, gain de maîtrise de soi.

L'autre apport d'importance souligné par les enseignants : le rôle du corps. «Je n'avais pas conscience de cela! » s'exclame Corinne Meynial. Le corps est le grand oublié de l'éducation. Pourtant, "nous vivons une époque sensationnelle» au sens propre du terme, reconnaissons-nous avec Anne Sauvageot (2003, p. 7). Le corps est objet de mille expériences, sollicitations, significations. " Porte-signe par excellence », il représente la "surface d'inscription de tous les textes sociaux (Brohm, 2001, p. 53). Combien de professions aujourd'hui dédiées au corps? De l'esthéticienne au chirurgien, de l'entraîneur sportif au coach de mise en forme, du styliste au fripier, du restaurateur au diététicien, etc. Le corps est partout, une abondante littérature lui est dédiée, sauf... en éducation à l'environnement où il devrait pourtant être le premier appelé. Certains praticiens s'en révoltent. Espinassous (2010, p. 10) raconte comment c'est « ce dehors qui [l]'a fait homme ». "Il faut se pencher sur ce que savent les corps » (Granger, 2009, p.132), et ce qu'ils savent faire; ceci d'autant plus urgemment que la virtualisation exponentielle des moyens de communication maintient nos corps assis tout en nous envoyant de façon dématérialisée dans n'importe quel lieu du cyberespace.

47 L'histoire des rapports hommes-nature, puis hommes-environnements, est une "trajection", c'est-à-dire un "processus qui dans le temps historique et l'espace géographique, accomplit et développe, en spirale pourrait-on dire, l'interaction entre la société et son environnement»(Berque, 1995, p. 37). Pouvons-nous choisir cette trajection? Pouvons-nous la guider? Pouvons-nous la décider collectivement? Et si nous décidons de le faire, quelle éducation des sens et du corps faut-il en déduire? «L'envie d'agir est bien moins commandée par le cerveau que par le corps, c'est un élan plus qu'une intention » souligne Anne Sauvageot (2003, p. 22). Construisons cet élan en repensant le corps dans l'éducation à l'environnement. 


\section{BIBLIOGRAPHIE}

Bachelard, G. (1960/1989). La poétique de la rêverie. Paris : Quadrige et PUF.

Berque, A. (1995). Les raisons du paysage. De la chine antique aux environnements de synthèse. Vanves : Hazan.

Brohm, J.-M. (2001). Le corps analyseur. Paris : Anthropos.

Centre d'Innovation et de Recherche en Pédagogie de Paris (CIRPP). Consulté le 10 mai 2012 sur http://cirpp.preau.ccip.fr/

Collectif (2005). Le Petit Larousse illustré (100e éd.). Paris : Larousse.

De Certeau, M. (1990). L'invention du quotidien. 1. arts de faire. Paris : Gallimard. Espinassous, L. (2010). Pour une éducation buissonnière. Saint-Claude-de-Diray : Hesse.

Godon N. (2008). Tout contre la pollution rationnelle : Dada, poésie sonore, Internationale situationniste. Écologie et politique, 36(2), 53-68.

Granger, C. (2009). Les corps d'été. Naissance d'une variation saisonnière, XXe siècle. Paris : Autrement.

Hall, E. T. (1971). La dimension cachée. Paris : Point / Seuil. Larousse (2011). Petit Larousse illustré.

Paris : Larousse.

Le Breton, D. (2006). La saveur du monde. Une anthropologie des sens. Paris : Métailié. Montagu, A.

(1979). La peau et le toucher. Un premier langage. Paris : Seuil.

Morin, E. et Kern, A.-B. (1993). Terre-Patrie. Paris : Seuil.

Moser, G. (2009). Psychologie environnementale. Les relations hommes - environnement. Bruxelles : de Boeck.

Paquot, T. (2006). Des corps urbains. Sensibilités entre béton et bitume. Paris : Autrement. Piaget, J. (1976). La formation du symbole chez l'enfant. Neuchâtel : Delachaux et Niestlé. Roustang, F. (2008). Savoir attendre. Pour que la vie change. Paris : Odile Jacob.

Sansot, P. (2004). Poétique de la ville. Paris : PBP / Payot et Rivages.

Sauvageot, A. (2003). L'épreuve des sens. De l'action sociale à la réalité virtuelle. Paris: PUF. Serres, M. (1985). Les cinq sens. Paris : Grasset et Fasquelle.

Wunenburger, J.-J. (1997). Philosophie des images. Paris : PUF.

\section{NOTES}

1. La formation qu'ils suivent mène au Brevet de technicien supérieur (BTS). En France, c'est un diplôme obtenu après deux ans de formation post-baccalauréat. Conçu à l'origine pour former des diplômés bac +2 opérationnels immédiatement sur le marché du travail, le BTS devient de plus en plus une opportunité de poursuite d'études. 


\section{RÉSUMÉS}

C'est d'abord par le corps que nous habitons le monde, "je sens donc je suis " dit Le Breton. Notre corps absorbe ou refuse, nous relie ou nous sépare, perçoit, se protège, se déplace, se tasse, réagit différemment en ville ou à la campagne, dans une rue escarpée ou une grande avenue, sur une plage ou dans une forêt, en un milieu inconnu ou un espace familier. Il est au cœur de notre saisie du monde. Et pourtant, grand paradoxe, en éducation à l'environnement, on ne lui accorde plus guère d'importance. On le maintient en salle de classe, il est immobile face à des expositions, limité aux manipulations d'outils, assis devant des ordinateurs. Cet article veut souligner ce paradoxe en s'appuyant sur une recherche-action menée dans le cadre d'une formation technique en aménagement paysager intégrant la pratique du land art.

It is at first through the body that we experience the world, "I feel therefore I am" said Le Breton. Our body absorbs or refuses, links or separates us, perceives, protects itself, moves, settles. It reacts differently being in the city or in the countryside, in a steep street or a main avenue, on a beach or in a forest, in an unknown environment or a familiar space. It is in the heart of our way of understanding the world. Paradoxically, we really don't pay attention to the body in environmental education. We maintain it in classrooms, immovable in front of exposures, restricted to tools manipulation, seated in front of computers. This article results from an action research project undertaken within a landscaping training program integrating land art practices.

\section{AUTEUR}

\section{DOMINIQUE COTTEREAU}

Docteure en sciences de l'éducation, occupe à mi-temps la fonction de consultante formatrice en EEDD au sein de la Scop Oxalis, Dominique Cottereau est maîtresse de conférences associée à l'IUT de Tours (Université François-Rabelais). Elle est également administratrice de l'association Echos d'Images (structure d'innovation pédagogique en ERE) et du Réseau d'éducation à

l'environnement en Bretagne, et chercheure pour le CIRPP. 